\title{
LSB HIDING USING RANDOM APPROACH FOR IMAGE WATERMARKING
}

\author{
Mayuri Verma ${ }^{1}$, Sheela Verma ${ }^{2}$ \\ ${ }^{I}$ M.E (CTA) Scholar, ${ }^{2}$ Assistant Professor,Department of Computer Science \& Engineering, SSGI, SSTC, Bhilai, \\ Chhattisgarh, India
}

\begin{abstract}
A digital image watermarking is the process of embedding an image with a secondary parameter called watermark, without deterioration in the quality of image to provide copyright protection means to provide protection for intellectual property from illegal copying. In this paper the method of nested digital image watermarking is used that means a watermark inside another watermark embedded into the cover image that is the main image. Here the Randomized LSB hiding algorithm is used for embedding one image into another as it has lesser complexity and the approach is more robust to the variations in the type of image. The blowfish algorithm is used to encrypt the watermark image before embedding into the cover image. The concept of encryption of watermark image before get embedded into the main image is used here to increase the security of the watermark image. This is because the research work is mainly focus on to get the more secured watermark by improving and enhancing the embedding capacity.
\end{abstract}

Key Words: Digital image Watermarking, Randomized LSB, Blowfish, Copyright Protection $* * *$

\section{INTRODUCTION}

Now a day's the Internet has become user friendly as it becomes an excellent distribution system for digital media (a form of electronic media where data are stored in digital form). Thus the, content owners see a high risk of piracy and concern about the protection of their intellectual property from illegal copying. Copyright protection of such data is an important issue.

Copyright protection of such multimedia data can be accomplished by the use of cryptographic algorithms which use to provide control over the accessing of data and make the data unreadable from the unauthorized users. But it is not possible to get the complete solution by using the cryptography algorithms. Thus the concept of digital watermarking was introduced which solves the problem of copyright of the intellectual property in digital media. It is used to identify the owner of the digital data.

The term watermark has been derived from the German term 'wassermarke' near about at the end of eighteenth century. The name 'Watermark' has been set because these marks have a similitude towards the upshot of water on paper [1]. Watermarking belong to a group of information hiding. In watermarking, the vital information is the 'external' data like images, voices etc... The 'internal' data that is watermark are the extra data for shielding the external data and to prove the ownership. The actual bits which represent the watermark should be scattered throughout the file in such a manner that they cannot be manipulated and identified.

\section{RELATED WORK}

There are lots of work have been proposed yet in the field of digital image watermarking for the purpose of getting security from illegal or unauthorized copyright of data. $\mathrm{P}$ Gupta [2] introduced the concept of Cryptography based Digital image watermarking algorithm to increase security of watermark data by using the blind watermarking technique which uses watermark nesting and encryption. For encryption XOR operation was used and DWT based technique used for embedding watermarked watermark in cover image. Further J S Bhalla and P Nagrath [3] had introduced the concept of Nested Digital Image Watermarking technique using blowfish algorithm. Here they mainly focused on increasing the embedding capacity and improving security of the watermarks by using LSB hiding technique for embedding.

One watermark is encrypted using the blowfish method and embedded into another watermark (using LSB Technique) and then this nested watermark is again encrypted and gets embedded into the main image. D Biswas, D Sarkar and A Pal [4] suggests that direct LSB hiding has drawback of having higher complexity and dependency on the type of image whereas Randomized LSB scores more in terms it has lesser complexity and is more robust to variations in the type of image by considering PSNR (Peak Signal to Noise Ratio). The research work by S P Singh and R Maini [5] provides a performance comparison between most of the common encryption algorithms (DES, 3DES, Blowfish, AES) which shows Blowfish has better performance than other encryption algorithms and it has no known security weak points so far. The comparison done on the basis of processing different sizes of data blocks by algorithm's to evaluate their encryption/decryption speed. LSB Based 
Lossless Digital Image Watermarking using Polynomials in Spatial Domain for DRM by A. Siva Sankar, T. Jayachandra Prasad and M.N. Giri Prasad [6] introduced a new embedding method where it randomly hide messages in the LSB of any component of the chosen pixel using polynomial thus probability of finding right coefficients correctly is difficult. Obaida Mohammad and Awad Al-Hazaimeh [7] proposed Hiding Data in Images Using New Random Technique. Here the message is inserted in the images in some random manner in the pixels of a cover image and LSB hides the message in a way that it is difficult to distinguish it. K M Singh, S B Singh and L S S Singh [8] proposes a least significant bit embedding algorithm for hiding encrypted messages in non-adjacent and random pixel locations in edges of the images. M O Ali and R Rao [9] proposed the basics of digital image watermarking and its hardware implementation. The blowfish algorithm is safer with respect to unauthorized attack and runs faster as compared to the other existing algorithms. The proposed algorithm was fabricate and realized using MATLAB by Pia Singh [10].

\section{SECURITY OF IMAGE AND COPYRIGHT PROTECTION}

In the literature review, it has been observed that the main focus emphasizes on improving the security of the watermarks by increasing the embedding capacity. The main aim of digital watermarking is to provide the copyright protection for the intellectual property which is in digital form. There are basically two challenges-How to keep illegal recipients (who do not have authority) from getting the copyrighted digital work.How to prevent illegal redistribution by rightful recipients of a copyrighted digital work.

The method of LSB hiding technique for embedding and extraction of watermark image was proposed earlier for nested digital image watermarking. But the direct LSB hiding technique has drawback of having higher complexity and dependency on the type of image whereas Randomized LSB hiding algorithm scores more in terms it has lesser complexity and is more robust to variations in the type of image with respect to the PSNR (Peak Signal to Noise Ratio) calculation. And also if the encryption could be done on only some selected pixels then it will provide more security as on the whole image.

\section{PROPOSED METHODOLOGY}

In this paper, the concept of nested digital image watermarking technique for embedding and extraction of watermark will be used. When one watermark image is embedded into another watermark image then it is known as nested watermark image. In the proposed method,

One watermark image is first encrypted using the blowfish algorithm, but the whole image is not encrypted here. Encryption will be done only on some selected pixels using
Key 1.

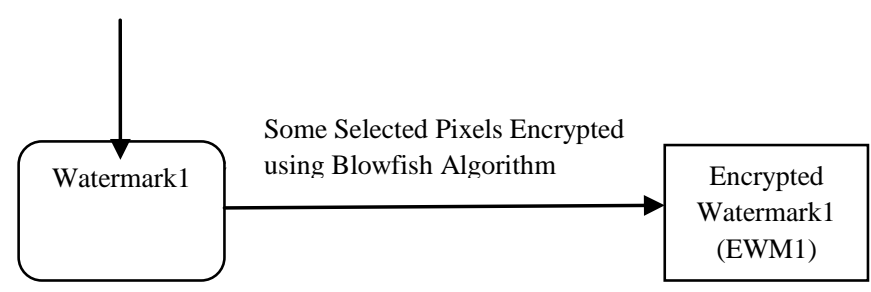

Fig -1: Encryption of first watermark image

Now the above encrypted watermark image (EWM1) gets embedded into another watermark using the Randomized mn LSB-LSB hiding algorithm using Key 2.

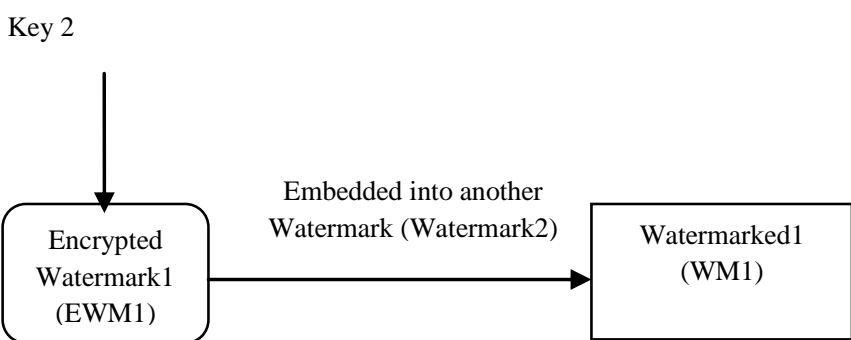

Fig -2: Encrypted watermark embedded into another watermark

This nested watermark WM1 (One watermark image embedded into another watermark image) is again gets encrypted using blowfish algorithm. Again the whole image is not encrypted; only some selected pixels get encrypted using Key 3.

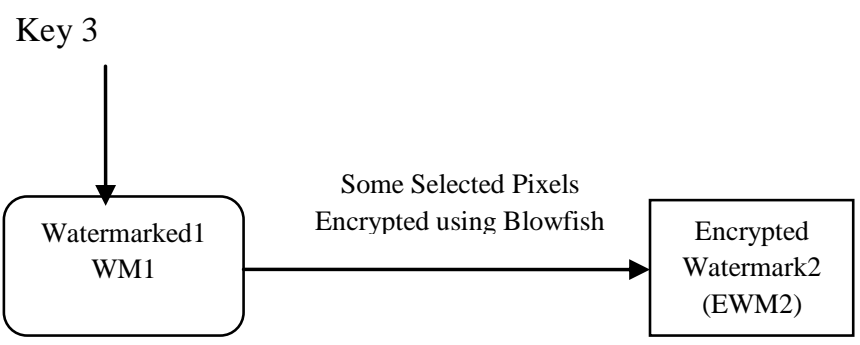

Fig -3: Encryption of Nested Watermark

Finally the above encrypted watermark image that is Encrypted Watermark2 (EWM2) is embedded into the main cover image using the Randomized m-n LSB-LSB hiding algorithm by using key 4 . 
Key 4

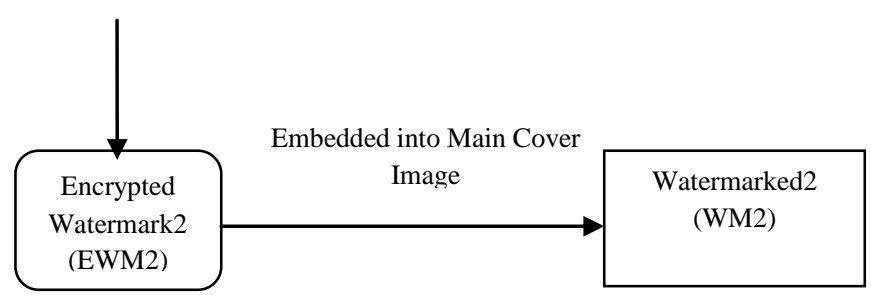

Fig -4: Watermark Embedding with Main Image

\subsection{Randomized m-n LSB-LSB Hiding Algorithm}

For embedding the watermark image, Randomized LSB hiding algorithm is used as it is having lesser complexity and its approach is more robust to variations in the type of image with respect to the PSNR (Peak Signal to Noise Ratio) calculation. [4] In LSB-LSB algorithm, the least significant bits of the original image gets masked and then substituted by the least significant bits of the watermark image. In randomized LSB hiding algorithm, ' $n$ ' represents the number of bits used (smaller the value of $n$, lesser will be the disturbance in the quality of image means degradation is quite invisible). In this method,

Generate $\mathrm{m}$ random numbers (which behaves as a secret key).

Apply $\mathrm{n}$ LSB-LSB hiding technique on some selected pixels, whose values are figure out by the secret keys.

Depending upon the values of ' $\mathrm{m}$ ', we apply LSB hiding in either rows or columns that is either horizontally or vertically.

This is the method of embedding watermark image using Randomized LSB hiding technique.

\section{EXPECTED OUTCOME}

In this paper, a new technique of digital image watermarking is introduced which provides more security to the images from unauthorized copying. The proposed technique increases the security by using the concept of nested digital watermarking. It applies the blowfish algorithm for encryption on some selected pixels which increases the security as it becomes unpredictable. Further embedding of watermark could be done using Randomized LSB Hiding technique, in which secret keys are random numbers and hiding technique applied on some selected pixels either in a row or in a column. Thus, the embedding capacity also improved by this technique. Therefore, with the proposed technique, it protects intellectual property from illegal copying by providing security.

\section{REFERENCES}

[1]Cox, I., Miller, M., Bloom, J. Digital Watermarking, Morgan Kaufmann, Ch 1, p. 6, Web ISBN-13: 978-0-08050459-9.

[2].Preeti Gupta, "Cryptography based digital image watermarking algorithm to increase security of watermark data", International Journal of Scientific \& Engineering
Research, Volume 3, Issue 9, September 2012 ISSN 22295518.

[3]. Jasdeep Singh Bhalla, Preeti Nagrath, "Nested Digital Image Watermarking Technique Using Blowfish Encryption Algorithm", International Journal of Scientific and Research Publications, Volume 3, Issue 4, April 2013 ISSN 22503153.

[4].D. Biswas, S. Biswas, P.P. Sarkar, D. Sarkar, S. Banerjee, A. Pal, "Comparison And Analysis Of Watermarking Algorithms In Color Images - Image Security Paradigm", International Journal of Computer Science \& Information Technology (IJCSIT), Vol 3, No 3, June 2011.

[5]. Simar Preet Singh, and Raman Maini, "Comparison Of Data Encryption Algorithms", International Journal of Computer Science and Communication Vol. 2, No. 1, January-June 2011, pp. 125-127.

[6]. A. Siva Sankar, T. Jayachandra Prasad, M. N. Giri Prasad, "LSB Based Lossless Digital Image Watermarking using Polynomials in Spatial Domain for DRM", 2nd International Conference and workshop on Emerging Trends in Technology (ICWET) 2011 Proceedings published by International Journal of Computer Applications (IJCA).

[7]. Obaida Mohammad and Awad Al-Hazaimeh, "Hiding Data in Images Using New Random Technique", IJCSI International Journal of Computer Science Issues, Vol. 9, Issue 4, No 2, July 2012, ISSN (Online): 1694-0814.

[8]. Kh. Manglem Singh, S. Birendra Singh and L. Shyam Sundar Singh, "Hiding Encrypted Message in the Features of Images", IJCSNS International Journal of Computer Science and Network Security, VOL.7 No.4, April 2007.

[9]. Mustafa Osman Ali and Rameshwar Rao, "Digital Image Watermarking Basics, and Hardware Implementation", International Journal of Modeling and Optimization, Vol. 2, No. 1, February 2012.

[10]. Pia Singh, "Image Encryption and Decryption Using Blowfish Algorithm in Matlab", International Journal of Scientific \& Engineering Research, Volume 4, Issue 7, July2013 ISSN 2229-5518.

\section{BIOGRAPHIES}

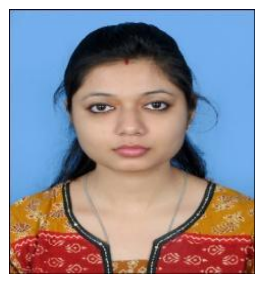

Mayuri Verma is a final year student of ME (CTA) of SSGI, SSTC, Bhilai, Chhattisgarh. She holds a bachelor's degree (BE) in Computer Science \& Engineering from Chhattisgarh Swami Vivekananda Technical University. Her area of interest in the field of research includes Cryptography \& Watermarking

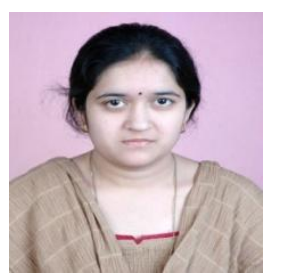

Sheela Verma is an Assistant Professor in Department of CSE in SSGI, SSTC, Bhilai, Chhattisgarh. She holds a Master's degree (ME) in Computer Technology \& Application and Bachelor's degree (BE) in CSE. She has 4 years of experience with area of specialization in the Field of Cryptography and field of Watermarking. 